\title{
THE INGRID PROJECT: DEVELOPMENT OF SOLUTIONS FOR SUSTAINABLE AND HIGHLY INTERCONNECTED GRIDS
}

\author{
Fabrizio D’Errico ${ }^{1}$, Adamo Screnci ${ }^{2}$, Massimo Bertoncini ${ }^{3}$ \\ ${ }^{1}$ Politecnico di Milano, Department of Mechanical Engineering, \\ Via La Masa 34, 20156 Milan (Italy) \\ ${ }^{2}$ Mc Phy Energy, 40 rue de Berges; 38000 Grenoble (France) \\ ${ }^{3}$ Engineering Ingegneria Informatica S.p.A., Via San Martino della Battaglia, 56 \\ 00185 Rome (Italy)
}

Keywords: Sustainable electricity, smart grids, energy career, hydrogen, energy storage

\begin{abstract}
One of the current main challenges in green-power storage and smart grids is the lack of effective solutions for accommodating the unbalance between renewable energy sources offering intermittent electricity supply - and a variable electricity demand. Integrating intermittent renewable energy sources by safe and cost-effective energy storage systems is today achievable. Coupled with electrolizers, high-capacity solid-state storage of green-hydrogen is practicable to sustain integration, monitoring and control of large quantity of GWh from renewable generation. The 23.9 MLN Euros INGRID European large demonstrative project started in July 2012 combines magnesium-based material solid-state hydrogen storage systems with advanced ICT technologies to intelligently interconnect miscellaneous energy networks (i.e. electricity and gas) and safely delivering green-hydrogen to various existing or forthcoming markets. One solution INGRID project addresses is an off-grid utility to store renewable electricity captured from wind sources to refill full-battery electric cars.
\end{abstract}

\section{Introduction}

The raw materials needed for energy supplies are taken from deposits which are not evenly distributed around the world and are, in many cases, located in regions affected by political instability. Globalization is leading to higher economic growth in successful emerging countries but also to more competition for fossil fuels. Relevant environmental and economic consequences of such a completion cannot be negligible today. Increasing of pollutant emissions as byproducts of power generation is high environmental price to sustain economic growth when largely based on combustion processes of fossil fuels. Over last decade, energy companies have been investing billions of euros in the expansion of power supply from renewable sources such as wind, water, solar, biomass and geothermal energy with some big challenges to face to compete with fossil fuels. It is known power generation plants using fossils as energy resources are normally built at sites which are conveniently located. Similarly to fossil fuels sources, renewable energy sources (RES) are available in certain areas. However, unlike fossil fuels, these energy sources are initially not linked to specific materials and cannot be simply transported and stored until they are required. Additionally, renewable energy sources are available when and where the wind blows or the sun shines; thus, power generation from RES subjected to variability and fluctuation. As a result, one major challenge power generation companies have been facing is to govern renewable energy peaks that cannot be accepted by grid for distribution (grid must be stabilized for their safe functioning). Despite wind farms would be economically competitive over their lifespan - considering both capital and operating 
expenditures at a whole - they suffer of highly fluctuant raw energy source, the wind. Peak loads cannot be accepted by grid, as above stated, thus wind farms are usually uneconomically managed as they are often totally or partially shut down. This fact results in a lot of power generation wasted, with negative impact onto unitary energy selling price.

Attempts are being made to avoid these problems by expanding power grids, at high investment costs and government centralized decisions on investments. On the other side, macro-trends moving toward new paradigm in the structure of the future energy system: energy management shall be more decentralized, more integrated on a local level with broadened application focus. Being respectful of operational security of the grid, the location of the new RES plants, mainly wind farms, would be determined by the owners and local authorities. On the other hand, situation for distribution is greatly complicated by small generator units widely distributed, since they would be often completely out of control (and sometimes even the observability) of the system operator. With large generator units, the system operator would be capable to manage multiple sources production. Unfortunately, in European system, Distributed Generation (DG) cannot dispatch energy as it likes, as the current system is driven by Transmission System Operators (TSO) which largely function within national boundaries. To promote change to distributed renewable energy generation, energy storage facilities would add more flexibility to the system. Especially in case of wind source, power generation is highly fluctuating with the time and often difficult to reliably predict. These facts make the planning and operation of the power system very difficult. In addition, DG is connected to the Low and Medium Voltage (LV and MV) grids. These systems have been planned to deliver electrical energy in a single direction, thus they have been designed to optimize the power flows from High Voltage (HV) stations to the loads. In this scenario, the introduction of minor amount of DG results in a reduced net loading of the transmission grid and it can be considered as a benefit. Nevertheless, a large amount of DG produces relevant effects on both distribution and transmission networks and it could dramatically impact on several technical frontlines. Over such scenario, energy storage solutions for intermittent highly available wind energy sources is a key for future energy securing in several EU Countries. Hydrogen batteries we discuss in the following here are one possible ready-to-market solution for large-capacity RES storage.

The storage opportunity of the hydrogen energy carrier

The production of hydrogen from water electrolysis fed by renewable intermittent sources represents a major long-term renewable energy storage opportunity. A number of benefits are provided to existing and new market applications. Potentially unlimited capacity of RES storage via-hydrogen conversion is key to unlock wind and solar distributed generation. An example of possible highly integrated supply-chain of RES power is shown in the scheme of Fig.1. The flow chart in Fig.1 considers numerous hydrogen advantages. Moreover in this scheme emerges how hydrogen can serve multiple energy uses with high integration. As Fig.1 illustrates, hydrogen produced by water electrolysis sourced by RES can be stored - in small or terawatt hour-scale quantities and in a variety of ways - can:

- $\quad$ Be used as centralized power generation, to be exploited by two possible pathways: a) use large scale hydrogen-compatible combined cycle gas turbines (CCGT), now under development ${ }^{1}$; b) use large-scale stationary fuel cell plants. Today the latter pathway is not a cost-effective solution, because of high cost of fuel cell technology

\footnotetext{
${ }^{1}$ By 2010 in Fusina (close to Venice, Italy), started demonstration plant consisting of a combined cycle in which a turbogas is fuelled with hydrogen in order to produce electricity and heat. The turbogas is equipped with a new conception burner specifically designed to be fuelled with pure hydrogen, and at the same time generate extremely low emissions of nitrogen oxide. The thermal energy produced by the combustion is converted into electricity in the gas turbine, reaching power which amounts to approximately $12 \mathrm{MW}$, whilst the flue gas are constituted exclusively of hot air and water vapour.
} 
and limited efficiency of reconversion of hydrogen into electricity. However much progresses are being in cost reduction and efficiency increase driven by rising up interest of some nations in this stationary power generation solution ${ }^{2}$.

- $\quad$ Be used as green-hydrogen for fuelling Fuel Cell Electric Vehicle (FCEV) fleet. Hydrogen produced from water electrolizers can be dispatched to the hydrogen refueling station close to the RES plant, piped to refueling station or transported to stations where pipeline network is not available ${ }^{3}$;

- Be supplied to industry as a "green" commodity or feedstock for current industrial uses, mainly petroleum and chemical industries. Currently 95\% of hydrogen is produced by reforming fossil fuel, thus employing processes that release lots of $\mathrm{CO} 2$;

- $\quad$ Be chemically combined with carbon to produce synthetic hydrocarbon fuels ${ }^{4}$ for transportation. Depending on process, the synthesis of hydrogen into hydrocarbon can produce synthetic natural gas (methane) or liquid synthetic fuel as methanol. As power is required to produce the synthetic products, either gas or liquid, and it is provided by the stored energy in hydrogen that is used for the specific synthesizing process, these concepts are referred in recent literature as power-to-gas and power-toliquid respectively. The most advantaged is the transportation and early exploitability issues: as synthetic methane is produced, it can be injected and stored in the existing gas grid as it is not distinguishable from fossil methane; furthermore it can be burned in conventional equipment to produce power and heat. Obviously, as the overall supply-chain is thought starting from the RES source, this option would allow to feed large quantity of power stored as renewable synthetic green-methane for sustainable mobility immediately available on market. Also the synthetic renewable methanol is ready for early exploitation as several fuel-flexible cars are taking advantages from methanol as clean-burning and easy-to-handle fuel.

- $\quad$ Be injected directly in the natural gas network, within the range 6-20\% renewably upgrade the carbon dioxide (CO2) fraction of biogas.

\footnotetext{
${ }^{2}$ In 2012 it was announced a series of large fuel cell installation 60 MW fuel cell park is under development by POSCO Energy along with partners in Hwaseong.

${ }^{3}$ Around the World there are a total of 80 hydrogen refueling stations (33 in Europe) supplying hydrogen produced by water electrolysis.

${ }^{4}$ Synthetic gas production specifically refers to methane following the reaction (1) $\mathrm{CO} 2+4 \mathrm{H} 2 \rightarrow \mathrm{CH} 4+2 \mathrm{H} 2 \mathrm{O}$ and the $\mathrm{CO}$ from sequestered $\mathrm{CO} 2$ following the reaction (2) $\mathrm{CO} 2+\mathrm{H} 2->\mathrm{CO}+\mathrm{H} 2 \mathrm{O}$. The hydrogen gas, together with CO produced by (2), is component of syngas (SNG) a valuable feedstock for the production of liquid fuel and other commodities. The production of SNG through hydrogen methanation also makes the recovery of CO2 for material use (e.g. in the chemicals sector) possible. The EU Emission Trading System (EU ETS), CO2 reduction goals, raw material scarcity, and price considerations are the major drivers for these markets.
} 


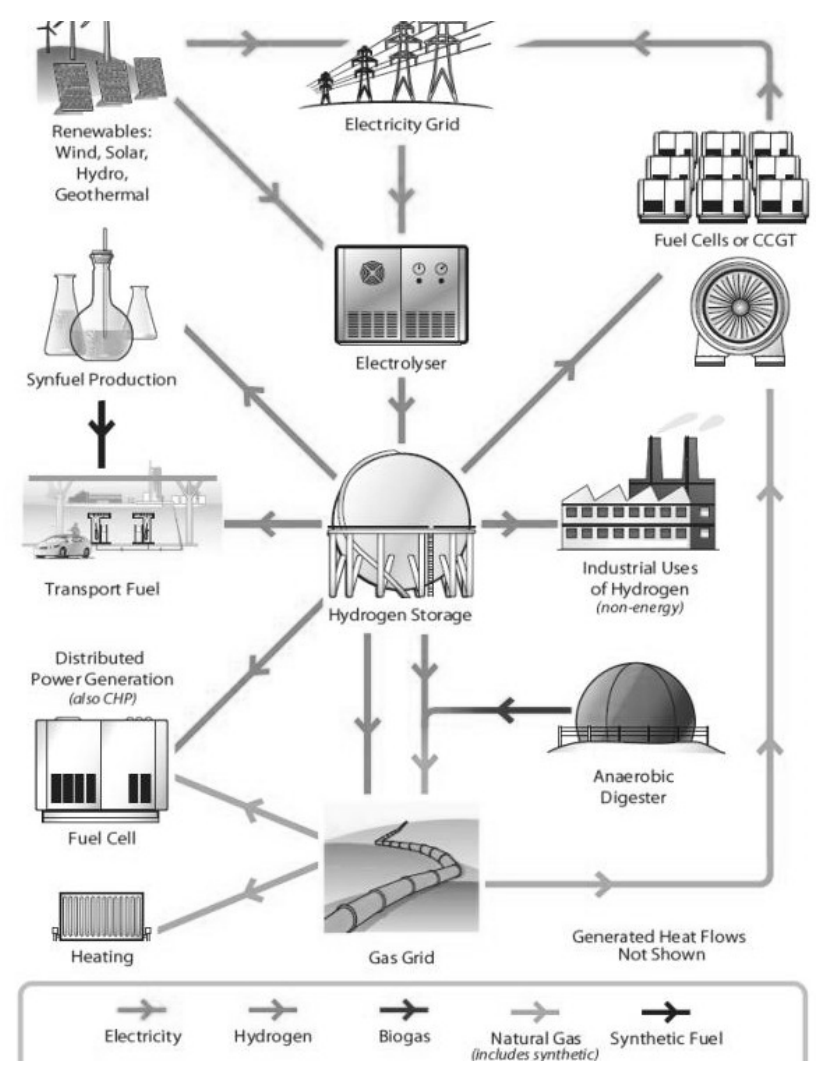

Figure 1 - The highly integrated energy network by water electrolysis RES-sourced (source: "Water electrolysis and Renewable Energy System“ released on May 2013 (latest check on June 2013 at website www.fuelcelltoday.com).

\section{The INGRID concept design and the real scenario}

The main scope of INGRID project is to realize in South of Italy a demonstrating plant capable to capture intermittent renewable generation produced by a wind farm located in such a highly congested local grid. Italy has witnessed a strong intermittency decentralized penetration over the past few years, as well as recurrent curtailments and congestion issues ${ }^{5}$. Decentralized generation causes more recurrent power flow inversions in High Voltage/Medium Voltage transformers (150/20 kV substations) as well as local high voltage. As negative consequence, high distributed RES generations causes large energy curtailment, a critical issue in Italy especially in such areas with weak transmission infrastructure and large supplying offer of renewable sources, such as Southern Italy. In such regions, as shown in the map of Fig.2, wind generation is highly developed. Wind curtailment represented $5.6 \%$ of the total wind generation in 2010 (470 GWh). In 2009, 10,7\% of the total wind generation was curtailed (around 700 GWh). In some regions, for example in the Southern Apennine Mountains, wind curtailment can reach up to $23 \%$ of total wind generation ${ }^{6}$.

\footnotetext{
${ }^{5}$ ENEL Distribuzione, partner of INGRID project and the main distributor system operator (DSO) in Italy, claims for 315,000 connections of decentralized generation in 2011, representing $19.5 \mathrm{GW}, 11.8 \mathrm{GW}$ of which of solar and 3.2 GW wind (Source, TERNA Report 2012).

${ }^{6}$ In 2013, the net generation was 289 TWh whereas the demand was 332 TWh: this deficit of 43 TWh $(12.9 \%$ of the demand) was covered through imports. Accordingly curtailment minimization is the major field requirement for INGRID energy storage service/application.
} 


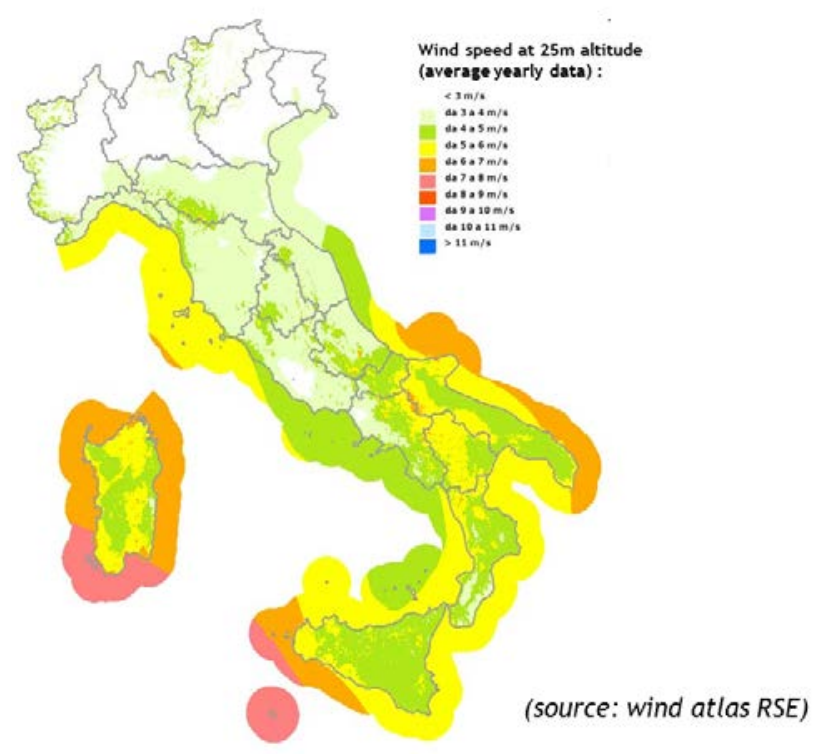

(a)

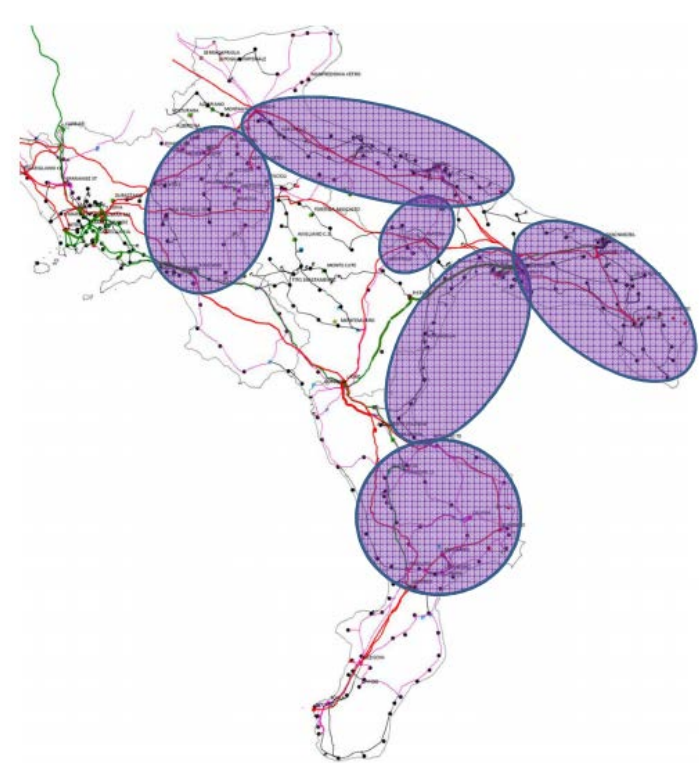

(b)

Fig. 2 - a) Italian wind source situation (RSE Wind Atlas); b) main critical patterns for evacuation of wind energy produced in the south of Italy (source: TERNA 2013)

The INGRID Project aims to design, construct and the operate a large energy storage demonstrator. A novel fast responding 1.2 MW electrolysis hydrogen generator coupled with safe hydrogen solid storage (HSS) units will provide smart balancing support for the local distribution in power grid. As main INGRID expected outcome, intermittent renewable energy sources available in wind farm will captured continuously, surpassing the problem of frequent shutting down due to distribution network congestion.

\section{The INGRID hardware architecture}

The INGRID plant rationale is depicted in Fig.3. As it is conceived, the plant aims to integrate intelligent monitoring, control and communication system with affordable and reliable technologies. About $1 \mathrm{GWh}$ is the order of stored electricity in INGRID plant. The plant is directly linked to medium voltage (MV) node close to local wind farm in city of Troia, in Apulia Region, South of Italy. The plant is designed to serve a local grid node by accepting power peaks generated by close wind farm. The Energy Management System, or EMS in the scheme of Fig.3, is the ICT intelligent device that can analyze multiple level information in order to manage (in real-time) distribution of electricity to INGRID generation plant, to by-pass partly or totally the RES power to the grid (i.e. weather forecast and statistical analysis for predictability of peak loads, real time market prices for hydrogen or electricity; etc. $)^{7}$. Water electrolizers (WE) in

\footnotetext{
${ }^{7}$ The Energy Management System (EMS) is the software stack which manages the INGRID node, and is in charge of decoupling intermittent RESs from power and gas distribution networks. It is the intelligent component of the INGRID system node, which will be in charge of managing and optimizing energy production, storage and control determining, by supervising and optimizing how much hydrogen should be produced with a view to flow out an optimized hydrogen/power amount. EMS main roles are to supervise and control power production from nearby RESs, manage power surplus stored as hydrogen and optimize energy delivery as hydrogen surplus through either a
} 
INGRID plant have been designed specifically for an instantaneous reply to such peak loads. Downstream to WE unit, the Green Energy Storage (GES) module realized by hydrogen solid storage modules (HSS) is the storing equipment of the plant. The hydrogen gas generated by WE unit flows into storage tanks that realizes the solid-state storing. As better described in the next section, gaseous hydrogen is absorbed by magnesium metal composite material, the basic material that constitutes the Hydrogen Solid Storage tanks (HSS). The hydrogen gas stored in HSS can therefore be employed when requested in several manners such as feeding fuel cells (FC) that equip the INGRID demonstrator for fueling a small fleet of battery electric vehicles (BEV), to supply hydrogen merchants directly by transporting the HSS tank modules by trucks.

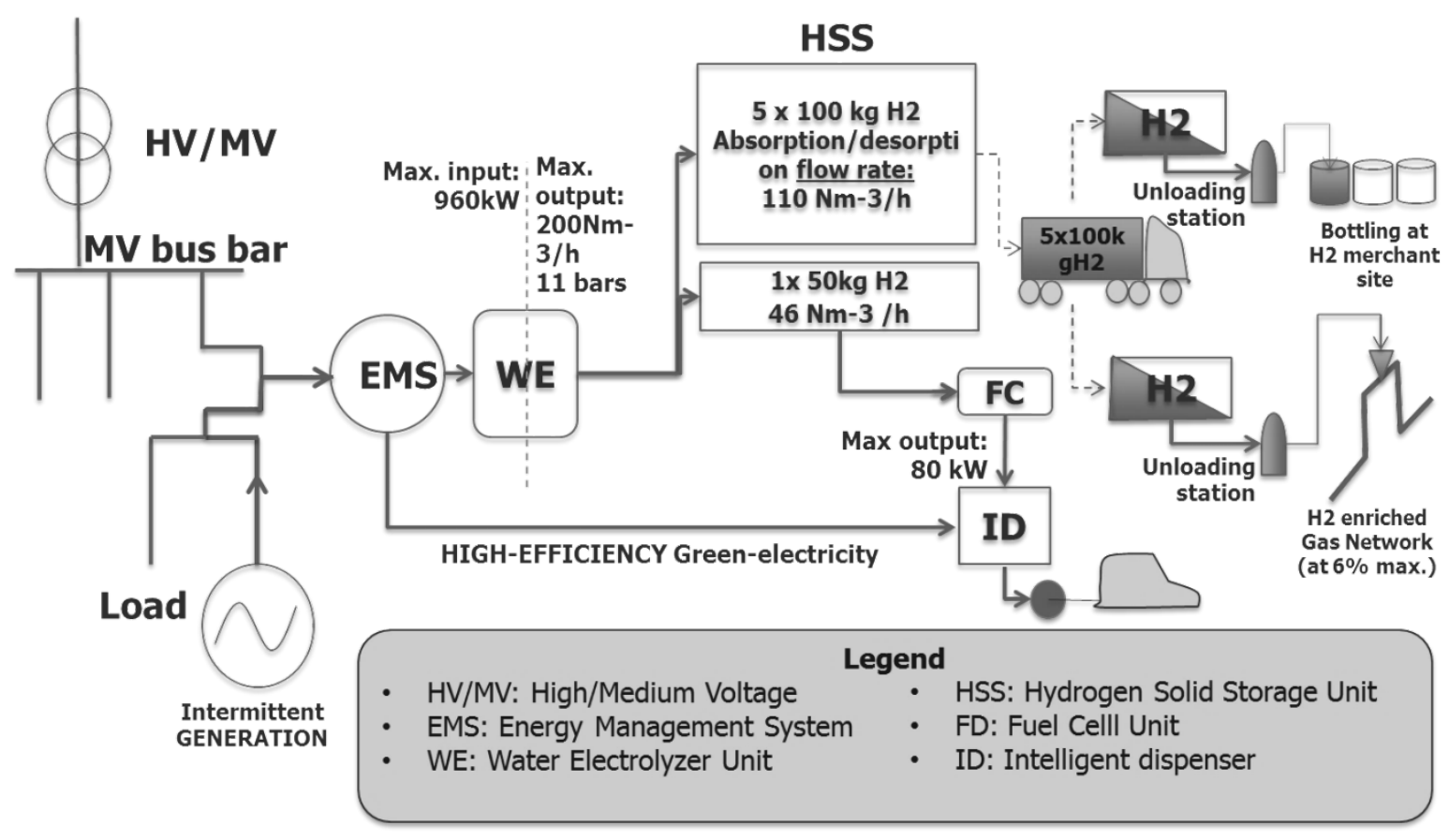

Fig. 3 - The INGRID demonstration site architecture as an example of the INGRID system solution.

Instantaneous response to peak load for the grid stabilizing

Water electrolizers and fuel cell stacks here employed have the major advantages of an instantaneous response to peak loads produced by wind farm. Highly fluctuant electricity produced by wind turbines flows into the water electrolyzer that use it to quite instantaneously produce hydrogen. Any request of electricity by downstream users (i.e. demand) is therefore decoupled from production (supplying) side thanks to water electrolizers and storage unit. More specifically, as shown in Fig.3, the INGRID demonstrative plant is directly connected to the Medium Voltage (MV) bar, where wind farm is connected. In such a real scenario, RES production cannot increase indefinitely at the MV access node of the grid, as such a high electricity variability cannot be managed by local grid. The INGRID plant is therefore structured to connect work with constant absorption of RES electricity in order to either reduce or increase power absorption - to produce less or more hydrogen - to smooth power peaks and stabilize the downstream grid's users. This approach allows to keep a high quality power curve also in case of large quantity of RES power plants connected. The Energy Management System (EMS) can decide the multiple-supply side basing on real-time information gathered by a multiple-demand

fuel cell serving local electric cars charging, either injecting hydrogen into a close gas network, or in a biomass plants, or in a gas turbine. 
requests (such as how much hydrogen shall be produced for the next hours? who are the multiple customers among hydrogen merchants, refilling stations, chemical industry to serve?) and profitability analysis (at which price could be bought by multiple customers hydrogen that we produce in next hours?) to produce less quantity of hydrogen (by reducing the power to electrolizers) and maximize the RES sourced electricity to the Intelligent Dispenser (ID) - refer to Fig. 3 - that links the EMS directly with the dispatching point of green-electricity.

\section{Magnesium metal as key-solution for safe and viable hydrogen solid storage}

Production, storage and dispatching of hydrogen as carbon-free green-energy carrier is technically allowed in INGRID plant by development of safe transportability of solid state hydrogen. Solid storage of gaseous hydrogen produced in WE is realized by use of high-capacity module made of composite material mainly based on magnesium metal. Magnesium metal is capable to store large quantity of hydrogen by absorbing atomic hydrogen to form solid compounds, namely the magnesium hydrides (MgH2), that are thermodynamically stable up to around $350^{\circ} \mathrm{C}$. Such a solid-state hydrogen storage method was selected as storage technology in INGRID project since it is intrinsically safe (no pressurized reactive hydrogen gas is present) and it is a very long-term storage. Regarding metallurgical phenomena involved, high diffusivity of hydrogen is recognized for several metals (hydrogen has a small atomic radius specie with high diffusivity in metal crystal lattice. Stability of solid compounds hydrogen can form with atomic metals as well as the reversibility of hydrogen atoms absorption (namely, the desorption reaction) are keys for deploying metals in hydrogen storing purposes. However, the kinetics of absorption and desorption phase is most relevant key issues to allow metal base material to be employed industrially as materials for hydrogen solid state storage. Among several metals to be employed for industrial applications, magnesium metal is one of most interesting materials, due to its low density and relative high storing capacity (when transportability of solid tanks is an issue $)^{8}$, rapid kinetics for absorption and desorption and relatively competitive costs. Specific researches have been conducted onto metallurgical issues to optimize kinetics for absorption and desorption to be fully compatible with industrial applications. To this scope, it is worth noticing hydrogen atoms penetrate and diffuse into metal by preferential routes; such routes are mainly grain boundaries because of their higher concentration of free spaces (vacations) left by magnesium atoms. Thus the "storing" phase develops preferentially through formation of intermetallic compounds at interstitial sites present in crystal lattice ${ }^{9}$. In last two decades further breakthroughs in hydrogen sorption kinetics - namely, solution to speed up the absorption and desorption phase - have been achieved by pre-treating magnesium by high-energy ball milling operations [1-5]. Ball milling mechanical treatment consists in applying severe plastic deformation to material to produce a highly refined microstructure, nanostructure, with a dramatic increase of grain boundaries, most preferred patterns for hydrogen diffusion. The importance of hydrogen kinetics sorption and lightness of hosting material (magnesium has very low density) are two key-issues to take into account for commercially exploiting solid-state hydrogen. Regarding to HSS unit specifically designed for INGRID plant, a minimum time for

\footnotetext{
${ }^{8}$ Magnesium has a good storage capacity, achieving theoretically the $7.6 \mathrm{wt} \%$ and it has density of $1.74 \mathrm{~g} / \mathrm{dm}^{3}$.

${ }^{9}$ Further constraints that work against absorption phase are: a) the presence of oxide layers that spontaneously formed in air on metals and, b) such a "barrier effect" that impedes hydrogen atoms to more deeply penetrate inside bulk because top layers saturate. These represents actually physical (oxide layers) and chemical (saturated layers) barriers that can prevent a fully hydrogen absorption inside bulk material; these barriers are generally responsible of lower sorption kinetics and practical absorption rate (weight percentage of hydrogen absorbed into host metal) far from theoretical values.
} 
complete loading/unloading operations of a 100kg capacity hydrogen module takes about 8 hours.

Additionally, one further drawback is due to the fact gaseous hydrogen can be absorbed by magnesium with an exothermic reaction, meanwhile the reversible desorption is an endothermic reaction. When hydrogen is absorbed and reacts with $\mathrm{Mg}$ to form $\mathrm{MgH} 2$, the reaction $\mathrm{Mg}+\mathrm{H} 2$ $\rightarrow \mathrm{MgH} 2$ releases heat. This heat shall be managed and thus evacuated. Conversely, the reaction of hydrogen desorption by magnesium $\mathrm{MgH} 2 \rightarrow \mathrm{Mg}+\mathrm{H} 2$ is endothermic, and this fact implies to provide to HSS module sufficient heat to start reaction and proceed. This heat energy have to be provided by external auxiliaries like electrical heating ${ }^{10}$. This facts lead to proper HSS module design to be safely handled, easily transported by truck and to allow rapid connection to auxiliaries for providing heating in the desorption stage. To satisfy all such technical constraints, the HSS module have been designed as show in Figure 4. Particularly, the single moveable standard tank container is realized with multiple of $100 \mathrm{~kg}$ hydrogen capacity HSS modules (Fig.4). Internally the HSS module are realized as detailed in Fig. 5, while in Fig.6 it is shown a schematic top view of the storing and charging area in INGRID plant. As it is visible, the HSS tanks are connected to hydrogen generation units (WE units) and when the HSS module is charged and ready for be transported to customer, it is linked to truck and moved. In the leftbottom part of Fig. 6 it is visible the fuel cell units that is directly connected to stationary solid storage module; in INGRID plant the FC unit serves for recharging BEV car locally available.

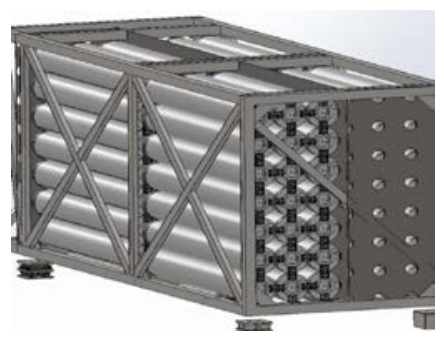

(a)

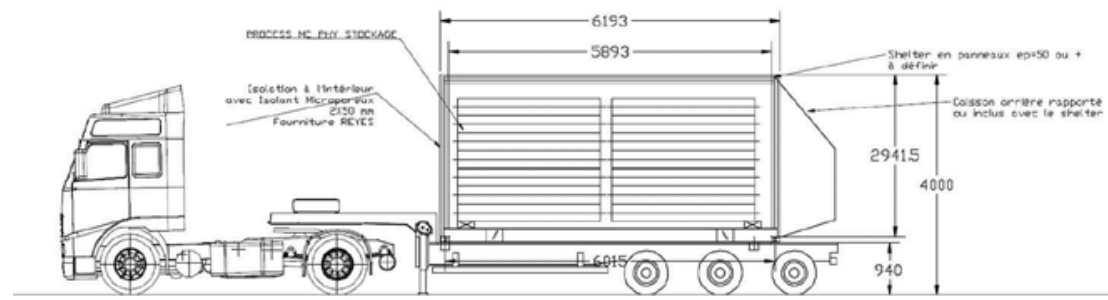

(b)

Figure 4 - Scheme of the transportable storage: a) the internal structure of HSS module, vessels for hydrogen storage are visible across metal frame; b) the HSS tank moveable container on truck.

\footnotetext{
10 The exothermic and endothermic reactions of hydrogen absorption and desorption respectively in magnesium lattice occurs around $300{ }^{\circ} \mathrm{C}$. One possible route to produce $\mathrm{Mg}$ hydride reactions with lowered reaction enthalpy (i.e. low temperatures for uptake and release phases) is to add elements which exhibit lower enthalpies of formation. $\mathrm{Mg} 2 \mathrm{Ni}$ is an example of this class of materials, as the enthalpies of formation for the ternary hydride Mg2NiH4 and the Mg2Ni is of the order of -67 kJmol-1 per H2, therefore lower than the $-78 \mathrm{kJmol}-1$ per H2 that is enthalpy for the MgH2 formation. Unfortunately this route reduces drastically the storage capacity and increases the density of the modified Mg-based material.
} 

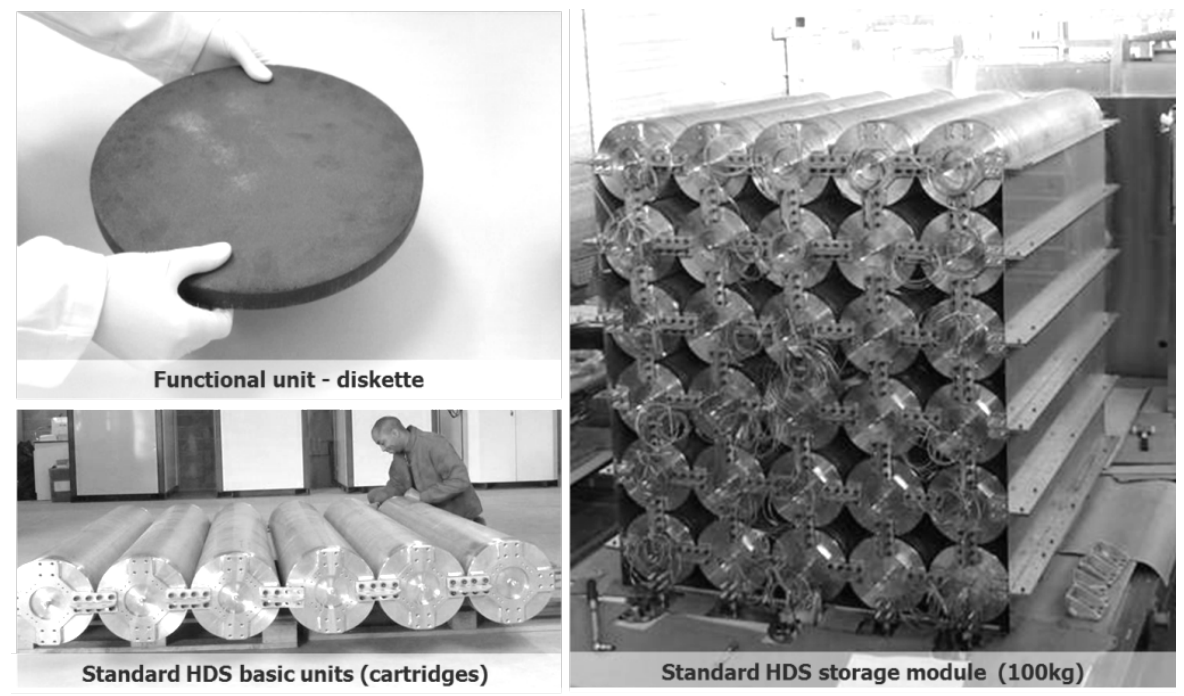

Figure 5 - Hydrogen solid storage module; anticlockwise view from up left: the single diskette module made of nanostructured magnesium; the single cartridge where several diskettes are placed; the 100kg module with several cartridges.

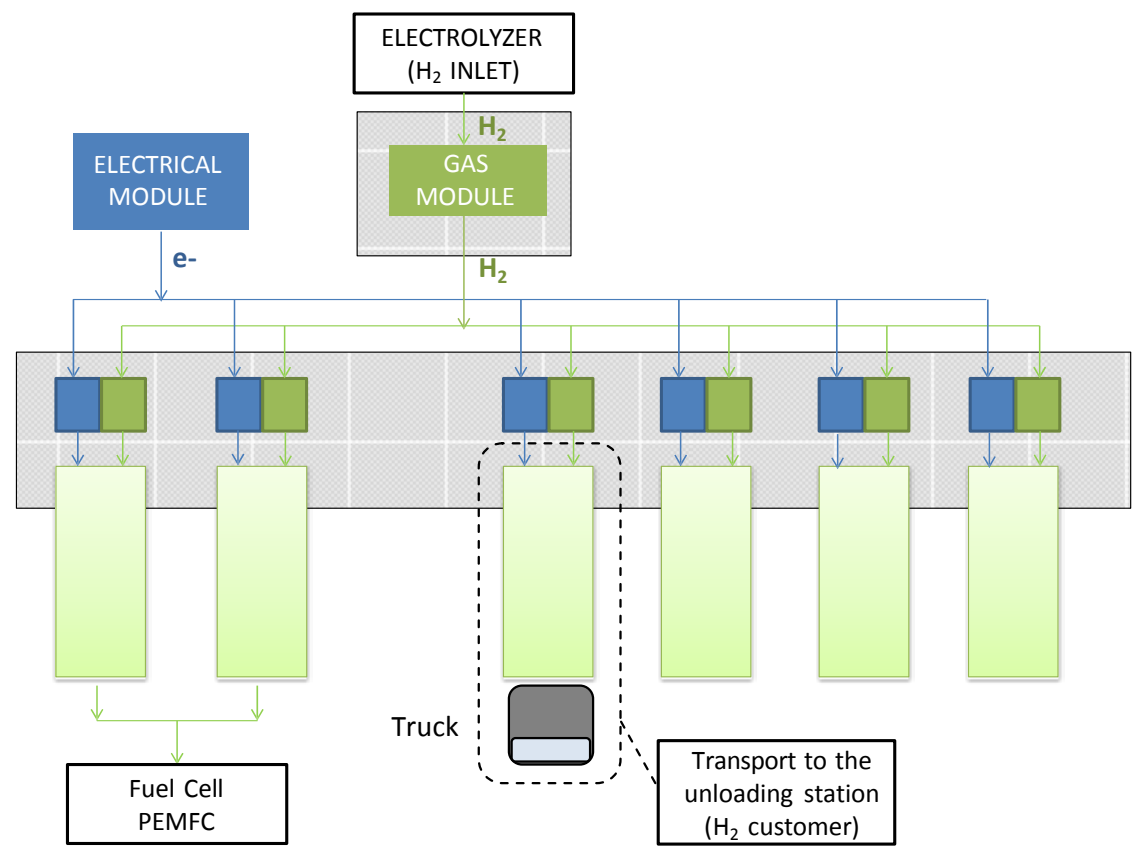

Figure 6 - Scheme of charging area in INGRID Plant.

\section{Conclusions}

The EU Commission Roadmap 2050 - Practical guide to a prosperous, low-carbon Europe (April 2010) states that the energy transition towards a decarbonized economy has benefits. This Roadmap has developed a reference projection of the energy transition to test different scenarios for technology and climate policies in the next half-century. Among those ones, lower energy costs per unit of output, more stable and predictable energy prices, an increased security of energy supply for much more economic stability, a more stable sustainable energy. In such a context, the INGRID concept can contribute in a concrete and fundamental portion in sustaining the European vision for this pathway. It is thought in order to building the pre-requisite conditions for the setting up of the EU "green energy economy from RES" in the near term 
period. Managing and deploying energy from RES help securing EU Countries from energy dependency on fossil fuel. By such a snapshot of the INGRID demonstrative case supply-chain, it is possible to verify in practice some key-issue, such as potentialities for interconnection of electricity grid and gas grid by exploiting green-hydrogen produced from excess of RES power.

\section{Acknowledgments}

This work makes use of results produced by the INGRID project (http://www.ingridproject.eu ), co-funded by the European Commission. Authors thank European Commission for supporting project dissemination activities. This work reflects only the authors' views. The Community is not liable for any use that may be made of the information contained therein.

\section{References}

1. G. Liang, J. Huot, S. Boily, A. van Neste and R. Schulz, Catalytic effect of transition metals on hydrogen sorption in nanocrystalline ball milled $\mathrm{MgH} 2-\mathrm{Tm}(\mathrm{Tm}=\mathrm{Ti}, \mathrm{V}, \mathrm{Mn}, \mathrm{Fe}$ and Ni) systems. J Alloys Compd, 292 (1999), p. 247-252

2. R. Shulz, J. Huot, G. Liang, S. Boily, G. Lalande and M.C. Denis, et al. "Recent developments in the applications of nanocrystalline materials to hydrogen technologies. Mater Sci Eng, A267 (1999), 240-245

3. A. Zaluska, L. Zaluski and J.O. Stroem-Olsen, "Structure, catalysis and atomic reactions on the nanoscale: a systematic approach to metal hydrides for hydrogen storage”, Appl Phys, A72 (2001), 157-165

4. R.C. Bowman and B. Fultz, Metallic hydrides: hydrogen storage and other gas-phase applications. MRS Bull, 27 (2002), 688-693

5. J. Huot, M. L. Tremblay and R. Schulz, "Synthesis of nanocrystalline hydrogen storage materials”, J Alloys Compd, 356-357 (2003), 603-607 